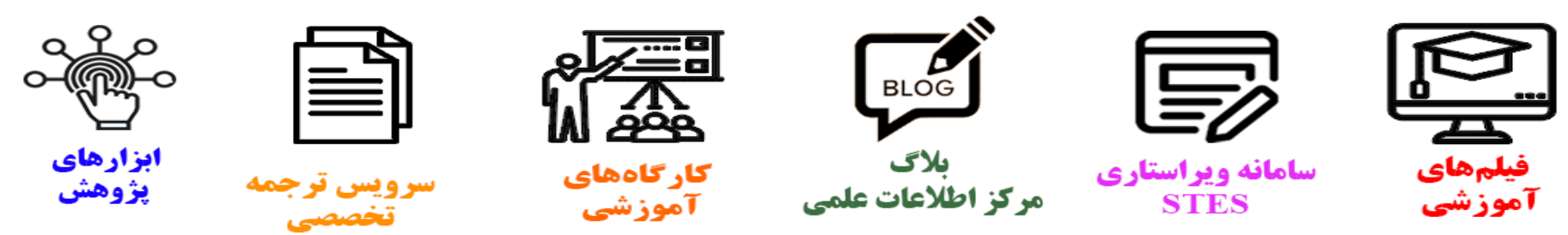

\title{
(c)
}

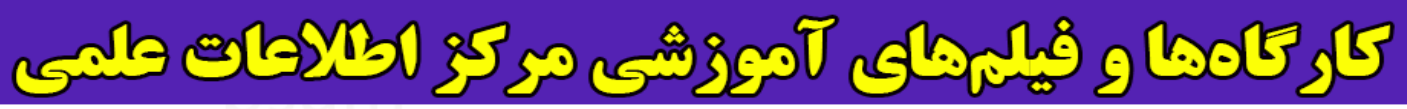
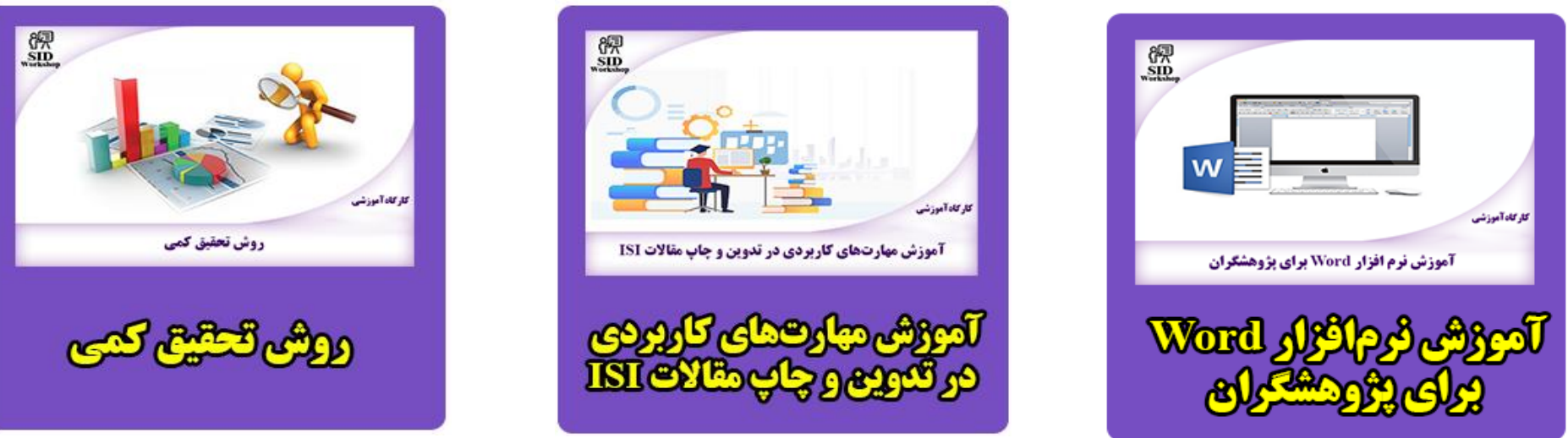


\title{
International Journal of Engineering
}

Journal Hom e page: ww w. i je.ir

\section{Thermal Convection in a (Kuvshiniski-type) Viscoelastic Rotating Fluid in the Presence of Magnetic Field through Porous Medium}

\author{
V. Kumar*a, P. Kumar \\ a Department of Mathematics, College of Engineering Studies, University of Petroleum \& Energy Studies (UPES), Dehradun-248007, \\ (Uttarakhand), India \\ ${ }^{b}$ Department of Mathematics, ICDEOL, Himachal Pradesh University, Shimla-171005, India
}

\section{PAPER INFO}

\section{Paper history:}

Received 22 July 2012

Received in revised form 10 December 2012

Accepted 28 February 2013

\section{Keywords:}

Thermal Convection

(Kuvshiniski-type) Viscoelastic Fluid

Magnetic Field

Rotation

Porous Medium

\section{$A B S T R A C A$}

The effect of magnetic field on an incompressible (Kuvshiniski-type) viscoelastic rotating fluid heated from below in porous medium is considered. For the case of stationary convection, magnetic field and medium permeability have both stabilizing and destabilizing effect on the thermal convection under some conditions whereas rotation has a stabilizing effect on the thermal convection. In the absence of rotation, medium permeability has a destabilizing effect while magnetic field has a stabilizing effect on the thermal convection in a Kuvshiniski viscoelastic rotating fluid. It is also found that presence of magnetic field and rotation introduce oscillatory modes in the system, whereas in their absence principle of exchange of stabilities is satisfied in the system. Graphs also have been plotted by giving some numerical values to the parameters.

doi: 10.5829/idosi.ije.2013.26.07a.10

\begin{tabular}{|c|c|c|c|}
\hline$d$ & Depth of layer, $[\mathrm{m}]$ & $k_{T}$ & Thermal diffusivity, $\left[\mathrm{m}^{2} / \mathrm{s}\right]$ \\
\hline a & Dimensionless wave number, $[-]$ & $v$ & Kinematic viscosity, $\left[\mathrm{m}^{2} / \mathrm{s}\right]$ \\
\hline g & Acceleration due to gravity, $\left[\mathrm{m} / \mathrm{s}^{2}\right]$ & $\lambda$ & Coefficient of viscoelasticity, \\
\hline$g$ & Gravity field, $\left[\mathrm{m} / \mathrm{s}^{2}\right]$ & $\rho$ & Density, $\left[\mathrm{kg} / \mathrm{m}^{3}\right]$ \\
\hline$k$ & Wave number, $[1 / \mathrm{m}]$ & $\nabla$ & Del operator, \\
\hline$k_{x}, k_{y}$ & Horizontal wave numbers, $[1 / \mathrm{m}]$ & $\partial$ & Curly operator, \\
\hline$n$ & Growth rate, $[1 / \mathrm{s}]$ & $D$ & Derivative with respect to $z(=d / d z)$ \\
\hline$Q$ & Chandrasekhar number, $[-]$ & \multicolumn{2}{|c|}{ Greek Symbols } \\
\hline$T_{A}$ & Taylor number, [-] & $\alpha$ & Coefficient of thermal expansion, $[1 / \mathrm{K}]$ \\
\hline$R$ & Modified Rayleigh number for porous medium, [-] & $\beta$ & Uniform temperature gradient, $[\mathrm{K} / \mathrm{m}]$ \\
\hline$T$ & Temperature, $[\mathrm{K}]$ & $T^{\prime}$ & Perturbation in temperature, $[\mathrm{K}]$ \\
\hline$t$ & Time, $[\mathrm{s}]$ & $p$ & Fluid pressure, $[\mathrm{pa}]$ \\
\hline $\boldsymbol{\Omega}(0,0, \Omega)$ & Rotation vector having components $(0,0, \Omega)$ & $p^{\prime}$ & Perturbation in pressure $p, \mathrm{psi}$ \\
\hline$H\left(h_{x}, h_{y}, h_{z}\right)$ & Magnetic field having components $\left(h_{x}, h_{y}, h_{z}\right)$ & $\rho^{\prime}$ & Perturbation in density $\rho$ \\
\hline$u, v, w$ & Component of velocity after perturbation & $\in$ & Medium porosity \\
\hline
\end{tabular}

*Corresponding Author Email: vivek_shrawat@yahoo.co.in; vivek.shrawat@gmail.com (V. Kumar) 


\section{INTRODUCTION}

The thermal instability of a fluid layer heated from below plays an important role in geophysics, oceanography, atmospheric physics, etc., and has been investigated by many authors, e.g., Be'nard [1], Rayleigh [2], Jeffreys [3]. A detailed account of the theoretical and experimental studies of so-called Be'nard convection in Newtonian fluids has been given by Chandrasekhar [4]. Bhatia and Steiner [5] have considered the effect of a uniform rotation on the thermal instability of a viscoelastic fluid and have found that rotation has a destabilizing influence, in contrast to the stabilizing effect on a Newtonian fluid. Sharma [6] has studied the thermal instability of a layer of Oldroydian fluid acted on by a uniform rotation and found that rotation has destabilizing and stabilizing effects under certain conditions.

The stability of flow of a single-component fluid through a porous medium taking into account the Darcy resistance has been considered by Lapwood [7] and Wooding [8]. The Darcy equation describes the incompressible flow of a Newtonian fluid of viscosity $\mu$ through a macroscopically homogeneous and isotropic porous medium of permeability $k_{1}$. If $q$ is the filter velocity of the fluid, the resistance term $-\left(\mu / k_{1}\right) q$ replaces the usual viscous term in the equations of fluid motion. There is mounting evidence, both theoretical and experimental, that suggests that Darcy's equation sometimes provides an unsatisfactory description of the hydrodynamic conditions, particularly near boundaries of a porous medium. Beavers et al. [9] have experimentally demonstrated the existence of shear within the porous medium near a surface where the porous medium is exposed to a freely flowing fluid, thus forming a zone of shear-induced fluid flow. Darcy's equation, however, cannot predict the existence of such a boundary zone, as no macroscopic shear term is included in this equation (Joseph and Tao [10]). To be mathematically compatible with the Navier-Stokes equations and physically consistent with the abovementioned experimentally observed boundary shear zone, Brinkman proposed the introduction of the term $-(\mu / \varepsilon) \nabla^{2} q$ in addition to $-\left(\mu / k_{1}\right) q$ in the equations of fluid motion. The elaborate statistical justification of the Brinkman equations has been presented by Saffman [11] and Lundgren [12].

With the growing importance of non-Newtonian fluids in technology and industries, the investigations of such fluids are desirable. Sharma et al. [13] have discussed the problem of thermosolutal instability of Rivlin-Ericksen rotating fluid in porous medium. The problem of thermosolutal convection in Rivlin-Ericksen fluid in porous medium in the presence of uniform vertical magnetic field and rotation is considered by Sharma et al. [14]. Sharma and Rana [15] have studied the problem of thermal instability of a Walters' (Model B') elastico-viscous fluid in a porous medium in the presence of variable gravity field and rotation.

Varshney and Dwivedi [16] have studied the unsteady effect on MHD free convection and mass transfer flow of a Kuvshiniski fluid through a porous medium with constant suction and constant heat and mass flux. Kumar and Singh [17] have studied the problem on a visco-elastic fluid heated from below in a porous medium and found that rotation has a stabilizing effect on the system and a Kuvshiniski visco-elastic fluid behaves like a Newtonian fluid in the problem.

Kumar and Kumar [18] have studied the problem on a couple-stress fluid heated from below in hydromagnetics and found that rotation has a stabilizing effect while magnetic field has both stabilizing and destabilizing effects on the system. The instability of the plane interface between two viscoelastic Kuvshiniski superposed fluids in porous medium in the presence of uniform rotation and variable magnetic field has been considered by Kumar [19]. Bishnoi and Goyal [20] have studied the problem of Soret Dufour driven thermosolutal instability of Darcy-Maxwell fluid and found that the Dufour number enhances the stability of Darcy-Maxwell fluid for stationary convection while it has a stabilizing character for overstability.

Keeping in mind the importance of non-Newtonian fluids, convection in a fluid layer heated from below, magnetic field and rotation, we propose to study the thermal convection in a (Kuvshiniski-type) viscoelastic rotating fluid in the presence of magnetic field through porous medium in the present paper.

\section{FORMULATION OF THE PROBLEM}

Consider a static state in which an incompressible, Kuvshiniski viscoelastic fluid layer of thickness $d$, is arranged, confined between two infinite horizontal planes situated at $Z=0$ and $z=d$, which is acted upon by a vertical magnetic field $\mathbf{H}(0,0, H)$, where $H$ is a constant, uniform rotation $\boldsymbol{\Omega}(0,0, \Omega)$ and variable gravity field $\mathbf{g}(0,0,-g)$. This fluid particle layer is assumed to be flowing through an isotropic and homogeneous porous medium of porosity $\in$ and medium permeability $k_{1}$. The fluid layer is heated from below leading to an adverse temperature gradient $\beta=\left(\left(T_{0}-T_{1}\right) / d\right)$, where $T_{0}$ and $T_{1}$ are the constant temperatures of the lower and upper boundaries with $T_{0}>T_{1}$. Let $p, \rho, T, \alpha, v, \lambda, k_{T}, \eta$ and $\mathbf{q}(u, v, w)$ denote respectively pressure, density, temperature, thermal 
coefficient of expansion, kinematic viscosity, coefficient of viscoelasticity, thermal diffusivity, electrical resistivity and velocity of the fluid. Following the Boussinesq approximations, the equations of motion, continuity and heat conduction of Kuvshiniski viscoelastic fluid in the presence of magnetic field (Kumar and Singh [17]) are:

$$
\begin{aligned}
& \frac{1}{\epsilon}\left(1+\lambda \frac{\partial}{\partial t}\right)\left[\frac{\partial \mathbf{q}}{\partial t}+\frac{1}{\epsilon}(\mathbf{q} . \nabla) \mathbf{q}\right]=-\frac{1}{\rho_{0}} \nabla p+\mathbf{g} \\
& \left(1+\frac{\delta \rho}{\rho_{0}}\right)+\left(1+\lambda \frac{\partial}{\partial t}\right)\left(\frac{v}{\epsilon} \nabla^{2}-\frac{v}{k_{1}}\right) \mathbf{q}+\left(1+\lambda \frac{\partial}{\partial t}\right) \\
& \frac{\mu_{e}}{4 \pi}[(\nabla \times \mathbf{H}) \times \mathbf{H}]+\frac{2}{\epsilon}\left(1+\lambda \frac{\partial}{\partial t}\right)(\mathbf{q} \times \Omega) \\
& \nabla . \mathbf{q}=0 \\
& \in \frac{d \mathbf{H}}{d t}=(\mathbf{H} \cdot \nabla) q+\in \eta \nabla^{2} \mathbf{H} \\
& \nabla . \mathbf{H}=0
\end{aligned}
$$

The equation of state is:

$$
\rho=\rho_{0}\left[1-\alpha\left(T-T_{0}\right)\right]
$$

where the suffix zero refers to value at the reference level $z=0$.

The equation of energy (Joseph [21]) is:

$E \frac{\partial T}{\partial t}+(q . \nabla) T=k_{T} \nabla^{2} T$

where $E=\epsilon+(1-\epsilon)\left(\rho_{s} c_{s} / \rho_{0} c\right)$ with $\rho_{0}, c, \rho_{s}, c_{s}$ denote the density and heat capacity of the fluid and the solid matrix, respectively. $v=\mu / \rho_{0}$ is the kinematic viscosity and $k_{T}=k / \rho_{0} c$ is the thermal diffusivity.

\section{BASIC STATE AND PERTURBATION EQUATIONS}

In the undisturbed state, the fluid is at rest. Constant temperatures are maintained in the fluid and uniform rotation and magnetic field act in the vertical direction (say in z-direction), therefore the basic state we wish to examine its stability is characterized by:

$$
\mathbf{q}=(0,0,0), \mathbf{\Omega}=(0,0, \Omega), \mathbf{H}=(0,0, \mathrm{H}), \mathrm{T}=\mathrm{T}_{0}-\beta \mathrm{z}
$$

with $\mathrm{T}=\rho=\rho(\mathrm{z}), \mathrm{p}=\mathrm{p}(\mathrm{z}) \mathrm{T}_{0}-\beta \mathrm{z}$ and

$\rho=\rho_{0}(1+\alpha \beta z)$

The character of equilibrium is examined by supposing that the system is slightly perturbed so that every physical quantity is assumed to be the sum of a mean and fluctuating component such that later is assumed to be very small in comparison to their equilibrium state values. Here, we assume that the small disturbances are functions of space and time variables. Let;

$(u, v, w)=\left(0+u^{\prime}, 0+v^{\prime}, 0+w^{\prime}\right),\left(h_{x}, h_{y}, h_{z}\right)=$

$\left(0+h_{x}^{\prime}, 0+h_{y}^{\prime}, H+h_{z}^{\prime}\right), T=T+T^{\prime}, \rho=\rho+\rho^{\prime}$,

$p=p+p^{\prime}$ be the quantities after perturbations in fluid velocity $\vec{q}(0,0,0)$, magnetic field $\vec{H}$, temperature T, density $\rho$ and pressure $\mathrm{p}$, where $u^{\prime}, v^{\prime}, w^{\prime}, T^{\prime}, \rho^{\prime} p^{\prime}$ are the perturbations in $u, v, w, T, \rho$ and $p$ respectively. After linearizing the perturbation equations and analyzing the perturbations into normal modes, we assume that the perturbation quantities are of the form

$$
\begin{aligned}
& {\left[\mathrm{w}, \mathrm{T}^{\prime}, \mathrm{h}_{\mathrm{z}}, \zeta, \xi\right]=[\mathrm{W}(\mathrm{z}), \Theta(\mathrm{z}), \mathrm{K}(\mathrm{z}), \mathrm{Z}(\mathrm{z}), \mathrm{X}(\mathrm{z})]} \\
& \exp \left\{\mathrm{ik}_{\mathrm{x}} \mathrm{x}+\mathrm{ik_{ \textrm {y } }} \mathrm{y}+\mathrm{nt}\right\}
\end{aligned}
$$

where $\mathrm{k}_{\mathrm{x}}$ and $\mathrm{k}_{\mathrm{y}}$ are the wave numbers in $\mathrm{x}$ and $\mathrm{y}$ directions, respectively and $\mathrm{k}=\sqrt{\mathrm{k}_{\mathrm{x}}^{2}+\mathrm{k}_{\mathrm{y}}^{2}}$ is the resultant wave number of propagation and $n$ is the frequency of any arbitrary disturbance which is, in general, a complex constant. $\zeta=\frac{\partial v}{\partial x}-\frac{\partial u}{\partial y}$ and $\xi=\frac{\partial h_{y}}{\partial x}-\frac{\partial h_{x}}{\partial y}$ are the z-components of the vorticity and current density, respectively. For the considered form of the perturbations in Equation (8), Equations (1) to (6) give:

$$
\begin{aligned}
& \frac{\mathrm{n}}{\epsilon}(1+\lambda \mathrm{n})\left(\mathrm{D}^{2}-\mathrm{k}^{2}\right) \mathrm{W}=-\mathrm{gk}^{2} \alpha \Theta+(1+\lambda \mathrm{n}) \\
& {\left[\frac{v}{\epsilon}\left(\mathrm{D}^{2}-\mathrm{k}^{2}\right)-\frac{v}{\mathrm{k}_{1}}\right]\left(\mathrm{D}^{2}-\mathrm{k}^{2}\right) \mathrm{W}+(1+\lambda \mathrm{n}) \frac{\mu_{\mathrm{e}} \mathrm{H}}{4 \pi \rho_{0}}} \\
& \left(\mathrm{D}^{2}-\mathrm{k}^{2}\right) \mathrm{DK}-\frac{2}{\epsilon}(1+\lambda \mathrm{n}) \Omega \mathrm{DZ} \\
& \frac{\mathrm{n}}{\epsilon}(1+\lambda \mathrm{n}) \mathrm{Z}=(1+\lambda \mathrm{n})\left[\frac{v}{\epsilon}\left(\mathrm{D}^{2}-\mathrm{k}^{2}\right)-\frac{v}{\mathrm{k}_{1}}\right] \mathrm{Z} \\
& +(1+\lambda \mathrm{n}) \frac{\mu_{\mathrm{e}} \mathrm{H}}{4 \pi \rho_{0}} \mathrm{DX}+\frac{2}{\epsilon}(1+\lambda \mathrm{n}) \Omega \mathrm{DW} \\
& \in \mathrm{nX}=\mathrm{H} \cdot \mathrm{DZ}+\in \eta\left(\mathrm{D}^{2}-\mathrm{k}^{2}\right) \mathrm{X} \\
& \in \mathrm{nK}=\mathrm{HDW}+\in \eta\left(\mathrm{D}^{2}-\mathrm{k}^{2}\right) \mathrm{K} \\
& \mathrm{En} \Theta=\beta \mathrm{W}+\mathrm{k}_{\mathrm{T}}\left(\mathrm{D}^{2}-\mathrm{k}^{2}\right) \Theta
\end{aligned}
$$

As both the boundaries are maintained at constant temperature, the perturbation in the temperature is zero and normal component of velocity must vanish at these boundaries. The appropriate boundary conditions are:

$$
W=0, \Theta=0 \text { at } z=0 \text { and } z=1
$$

Here, we consider the case of two free boundaries, where the tangential stresses vanish and therefore we have: 
$\frac{\partial^{2} W}{\partial z^{2}}=0 \quad$ at $\quad z=0$ and $\quad z=1$

On using the non-dimensional parameter $\mathrm{a}=\mathrm{kd}$, $\sigma=\frac{\mathrm{nd}^{2}}{v}, \mathrm{p}_{1}=\frac{v}{\mathrm{k}_{\mathrm{T}}}, \mathrm{p}_{2}=\frac{v}{\eta}, \quad \mathrm{p}_{\mathrm{e}}=\frac{\mathrm{k}_{1}}{\mathrm{~d}^{2}}, \quad \mathrm{~F}=\frac{\lambda \nu}{\mathrm{d}^{2}} \quad$ and $\mathrm{D}^{*}=\mathrm{dD}$ and dropping $(*)$ for convenience and eliminating the physical quantities $\Theta, Z, X$ and $K$, the final stability governing equation is obtained as:

$$
\begin{aligned}
& (1+\sigma F)\left[\sigma-\left\{\left(D^{2}-a^{2}\right)-\frac{\epsilon}{p_{e}}\right\}\right]\left[\left\{\sigma-\left\{\left(D^{2}-a^{2}\right)\right.\right.\right. \\
& \left.\left.\left.-\frac{\epsilon}{p_{e}}\right\}\right\}\left(D^{2}-a^{2}-\sigma p_{2}\right)+Q D^{2}\right]\left(D^{2}-a^{2}-E \sigma p_{1}\right) \\
& \left(D^{2}-a^{2}-\sigma p_{2}\right)\left(D^{2}-a^{2}\right) W-R a^{2} \\
& {\left[\left\{\sigma-\left\{\left(D^{2}-a^{2}\right)-\frac{\epsilon}{p_{e}}\right\}\right\}\left(D^{2}-a^{2}-\sigma p_{2}\right)+Q D^{2}\right]} \\
& {\left[D^{2}-a^{2}-\sigma p_{2}\right] W+Q(1+\sigma F)\left[\left\{\sigma-\left\{\left(D^{2}-a^{2}\right)\right.\right.\right.} \\
& \left.\left.\left.-\frac{\epsilon}{p_{e}}\right\}\right\}\left(D^{2}-a^{2}-\sigma p_{2}\right)+Q D^{2}\right]\left(D^{2}-a^{2}-E \sigma p_{1}\right) \\
& \left(D^{2}-a^{2}\right) D^{2} W+T_{A}(1+\sigma F)\left(D^{2}-a^{2}-\sigma p_{2}\right)^{2} \\
& \left(D^{2}-a^{2}-E \sigma p_{1}\right) D^{2} W=0
\end{aligned}
$$

Here, $\quad R=\left(g \in \alpha \beta d^{4} / v k_{T}\right)$ is the modified Rayleigh number for porous medium, $T_{A}=\left(4 \Omega^{2} d^{4} / v^{2}\right)$ is the Taylor number and $Q=\left(\mu_{e} H^{2} d^{2} / 4 \pi \rho_{0} v \eta\right)$ is the Chandrasekhar number. The boundary conditions (14) and (15) for the problem transform to:

$$
W=0, D^{2} W=0 \quad \text { at } z=0 \text { and } \quad z=1
$$

The proper solution of Equation (17) characterizing the lowest mode is:

$W=W_{0} \sin \pi z$

where $W_{0}$ is constant. Substituting Equation (18), Equation (16) gives:

$$
\begin{aligned}
& R_{1}=\frac{(1+\sigma F)(1+x)\left[i \sigma_{1}+\left(1+x+\frac{\epsilon}{p_{e}}\right)\right]}{x}\left(1+x+i \sigma_{1} E p_{1}\right)+\frac{Q_{1}(1+\sigma F)(1+x)\left(1+x+i \sigma_{1} E p_{1}\right)}{x\left(1+x+i \sigma_{1} p_{2}\right)} \\
& +\frac{T_{A}(1+\sigma F)\left(1+x+i \sigma_{1} E p_{1}\right)\left(1+x+i \sigma_{1} p_{2}\right)}{x\left\{\left[i \sigma_{1}+\left(1+x+\frac{\epsilon}{p_{e}}\right)\right]\left[1+x+i \sigma_{1} p_{2}\right]+Q_{1}\right\}}
\end{aligned}
$$

where

$$
R_{1}=\frac{R}{\pi^{4}}, i \sigma_{i}=\frac{\sigma}{\pi^{2}}, P=\pi^{2} P_{\mathrm{e}}, T_{A}=\frac{T_{A}}{\pi^{4}}, x=\frac{a^{2}}{\pi^{2}}
$$

\section{RESULTS AND DISCUSSION}

\section{1. Stationary Convection}

For the case of stationary convection, when the instability sets, the marginal state will be characterized by $\sigma=0$. Thus, putting $\sigma=0$ in Equation (19), we get:

$$
\begin{aligned}
& R_{1}=\frac{(1+x)}{x}\left[\left(1+x+\frac{\epsilon}{P}\right)(1+x)+Q_{1}\right]+T_{A_{1}}(1+x) \\
& \left.\left\{\left(1+x+\frac{\epsilon}{P}\right)(1+x)+Q_{1}\right\}^{-1}\right]
\end{aligned}
$$

which express the Rayleigh number $R_{1}$ as a function of the parameters $Q_{1}, T_{A}, P$ and dimensionless wave number $X$. Here, the viscoelastic parameter $F$ vanishes with $\sigma$ and thus Kuvshiniski fluid behaves like an ordinary Newtonian fluid. Further, to study the effect of magnetic field, rotation and medium permeability, we study the behavior of $d R_{1} / d Q_{1}, d R_{1} / d T_{A}$ and $d R_{1} / d P$ analytically.

From Equation (20), we have:

$$
\frac{d R_{1}}{d Q_{1}}=\frac{(1+x)}{X}\left[1-\frac{T_{A_{1}}(1+x)}{\left[(1+x)\left(1+x+\frac{\epsilon}{P}\right)+Q_{1}\right]^{2}}\right]
$$

which shows that magnetic field has stabilizing/destabilizing effect on the thermal convection in a Kuvshiniski visco-elastic fluid under the conditions

$$
\begin{aligned}
& T_{A}(1+x)<\left[(1+x)\left(1+x+\frac{\epsilon}{P}\right)+Q_{1}\right]^{2} \\
& \text { Or } T_{A}(1+x)>\left[(1+x)\left(1+x+\frac{\epsilon}{P}\right)+Q_{1}\right]^{2} .
\end{aligned}
$$

But for the permissible values of various parameters, the said effect is stabilizing only if

$T_{A_{1}}(1+x)<\left[(1+x)\left(1+x+\frac{\epsilon}{P}\right)+Q_{1}\right]^{2}$

In the absence of rotation $\left(T_{A}=0\right)$, Equation (21) becomes:

$\frac{d R_{1}}{d Q_{1}}=\frac{(1+x)}{x}$

which clearly shows that magnetic field has a stabilizing effect on thermal convection in a Kuvshiniski viscoelastic fluid through porous medium in the absence of rotation. From Equation (20), we have

$$
\frac{d R_{1}}{d T_{A_{1}}}=\frac{(1+x)^{2}}{x\left[(1+x)\left(1+x+\frac{\epsilon}{P}\right)+Q_{1}\right]}
$$


which clearly shows that rotation has a stabilizing effect on thermal convection in a Kuvshiniski visco-elastic fluid through porous medium.

From Equation (20), we have:

$\frac{d R_{1}}{d P}=\frac{(1+x)^{2} \in}{P^{2} x}\left[T_{A_{1}}(1+x)\left\{(1+x)\left(1+x+\frac{\epsilon}{P}\right)+Q_{1}\right\}^{-2}-1\right]$

which shows that medium permeability has stabilizing/destabilizing effect on thermal convection in a Kuvshiniski visco-elastic fluid under the conditions

$$
\begin{aligned}
& T_{A_{1}}(1+x)>\left[(1+x)\left(1+x+\frac{\epsilon}{P}\right)+Q_{1}\right]^{2} \\
& \text { or } T_{A_{1}}(1+x)<\left[(1+x)\left(1+x+\frac{\epsilon}{P}\right)+Q_{1}\right]^{2} .
\end{aligned}
$$

But for the permissible values of various parameters, the medium permeability has a stabilizing effect contrary to its general destabilizing influence if

$$
T_{A_{1}}(1+x)>\left[(1+x)\left(1+x+\frac{\epsilon}{P}\right)+Q_{1}\right]^{2}
$$

In the absence of rotation $\left(T_{A_{4}}=0\right)$, Equation (24) becomes:

$$
\frac{d R_{1}}{d P}=-\frac{(1+x)^{2} \in}{x P^{2}}
$$

which confirms that medium permeability has a destabilizing effect on thermal convection in a Kuvshiniski visco-elastic fluid through porous medium in the absence of rotation as derived by Kumar and Singh [17].

In the absence of magnetic field $\left(Q_{1}=0\right)$, Equation (24) becomes:

$$
\frac{d R_{1}}{d P}=\frac{(1+x)^{2} \in}{P^{2} X}\left[\frac{T_{A_{1}}}{(1+X)\left(1+X+\frac{\epsilon}{P}\right)^{2}}-1\right]
$$

which shows that medium permeability has stabilizing/destabilizing effect on thermal convection in a Kuvshiniski visco-elastic fluid in the absence of magnetic field (as derived by Kumar and Singh [17]) under the conditions

$$
T_{A_{1}}>\text { or }<(1+x)\left(1+x+\frac{\epsilon}{P}\right)^{2} \text {. }
$$

\section{2. Stability of the System and Oscillatory} Modes Multiplying Equation (9) by $W^{*}$ and integrating over the range at $Z$ and making use of Equations (10) to (13) with the Equation (8) and boundary conditions (17), we get:

$$
\begin{aligned}
& (1+\sigma F)\left(\frac{\sigma}{\epsilon}+\frac{1}{p_{e}}\right) I_{1}+\frac{(1+\sigma F)}{\epsilon} I_{2}-\frac{g \alpha k_{T} a^{2}}{v \beta} \\
& {\left[I_{3}+\sigma^{*} E p_{1} I_{4}\right]+(1+\sigma F) \frac{\mu_{e} \in \eta}{4 \pi \rho_{0} v}\left[I_{5}+\sigma^{*} p_{2} I_{6}\right]} \\
& +d^{2}(1+\sigma F) \in\left\{\left(\frac{\sigma^{*}}{\epsilon}+\frac{1}{p_{e}}\right) I_{7}+\frac{1}{\epsilon} I_{8}\right\}+ \\
& (1+\sigma F) \frac{\mu_{e} \in \eta d^{2}}{4 \pi \rho_{0} v}\left[I_{9}+\sigma p_{2} I_{10}\right]=0
\end{aligned}
$$

where

$$
\begin{aligned}
& I_{1}=\int\left(|D W|^{2}+a^{2}|W|^{2}\right) d z, \\
& I_{2}=\int\left(\left|D^{2} W\right|^{2}+2 a^{2}|D W|^{2}+a^{4}|W|^{2}\right) d z, \\
& I_{3}=\int\left(|D \Theta|^{2}+a^{2}|\Theta|^{2}\right) d z, I_{4}=\int|\Theta|^{2} d z, \\
& I_{5}=\int\left(\left|D^{2} K\right|^{2}+a^{4}|K|^{2}+2 a^{2}|D K|^{2}\right) d z, \\
& I_{6}=\int\left(|D K|^{2}+a^{2}|K|^{2}\right) d z, I_{7}=\int|Z|^{2} d z, \\
& I_{8}=\int\left(|D Z|^{2}+a^{2}|Z|^{2}\right) d z, \\
& I_{9}=\int\left(|D X|^{2}+a^{2}|X|^{2}\right) d z, I_{10}=\int|X|^{2} d z
\end{aligned}
$$

where $\sigma^{*}$ is the complex conjugate of $\sigma$. All the integrals $I_{1}$ to $I_{10}$ are positive definite. Putting $\sigma=i \sigma_{i}$ in Equation (27) and equating the imaginary parts, we obtain:

$$
\begin{aligned}
& \sigma_{i}\left[\left(\frac{1}{\epsilon}+\frac{F}{p_{e}}\right) I_{1}+\frac{F}{\epsilon} I_{2}+\frac{g \alpha k_{T} a^{2}}{v \beta} E p_{1} I_{4}+\frac{\mu_{e} \eta}{4 \pi \rho_{0} v}\right. \\
& \left\{F I_{5}-p_{2} I_{6}\right\}+d^{2}\left\{\left(\frac{F}{p_{e}}-\frac{1}{\epsilon}\right) I_{7}+\frac{F}{\epsilon} I_{8}\right\} \\
& \left.+\frac{\mu_{e} d^{2} \in \eta}{4 \pi \rho_{0} v}\left\{F I_{9}+p_{2} I_{10}\right\}\right]=0
\end{aligned}
$$

In the absence of magnetic field and rotation, Equation (28) becomes:

$\sigma_{i}\left[\left(\frac{1}{\epsilon}+\frac{F}{p_{e}}\right) I_{1}+\frac{F}{\epsilon} I_{2}+\frac{g \alpha k_{T} a^{2}}{v \beta} E p_{1} I_{4}\right]=0$

From Equation (29), it is obvious that all the terms in the bracket are positive definite. Thus, $\sigma_{i}=0$ which means that oscillatory modes are not allowed in the system and principle of exchange of stabilities (PES) is satisfied in the absence of magnetic field and rotation in the system. So, we can say that oscillatory modes are introduced due to the presence of magnetic field and rotation.

\section{NUMERICAL COMPUTATION}

Now, the critical thermal Rayleigh number for the onset of instability is determined numerically using NewtonRaphson method by the condition $d R_{1} / d x=0$. As a 
function of $x, R_{1}$ is given by Equation (20) which attains its minimum when $d R_{1} / d x=0$ with $x$ determined as a solution of equation by putting $d R_{1} / d x=0$ in powers of $x$. Equation (20) will give the required critical thermal Rayleigh number $R_{l}$ for various values of critical wave number $X$. In Figure 1 and Figure 2, the critical Rayleigh number $R_{1}$ increases with increase in magnetic field parameter $Q_{1}$ which shows that magnetic field has a stabilizing effect on the thermal convection. In Figure 3 and Figure 4, the critical Rayleigh number $R_{1}$ increases with increase in rotation parameter $T_{A}$ which shows that rotation has a stabilizing effect on the thermal convection. In Figure 5, the critical Rayleigh number $R_{1}$ decreases with increase in medium permeability $P$ which shows that medium permeability has a destabilizing effect on the thermal convection. In Figure 6 and Figure 7, the critical Rayleigh number $R_{1}$ increases with increase in medium permeability $P$ which shows that medium permeability has a stabilizing effect on the thermal convection for the permissible range of values of various parameters.

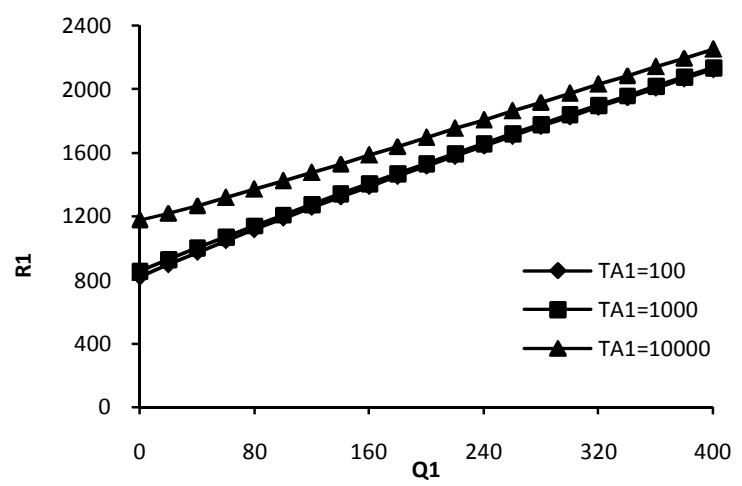

Figure 1. Variation of critical Rayleigh number $R_{l}$ with magnetic field $Q_{1}$ for a fixed $\epsilon=0.5, P=0.005$ and $T_{\text {A }}=100,1000,10000$.

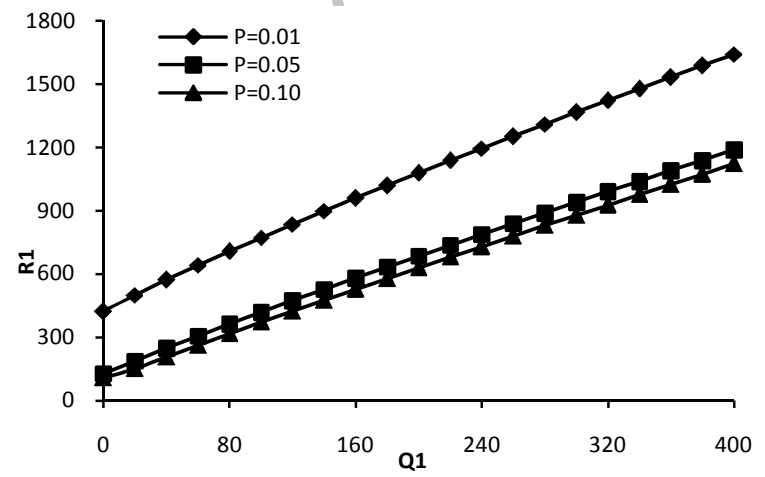

Figure 2. Variation of critical Rayleigh number $R_{1}$ with magnetic field $Q_{1}$ for a fixed $\in=0.5, T_{A_{1}}=100$ and $P=0.01,0.05,0.10$.

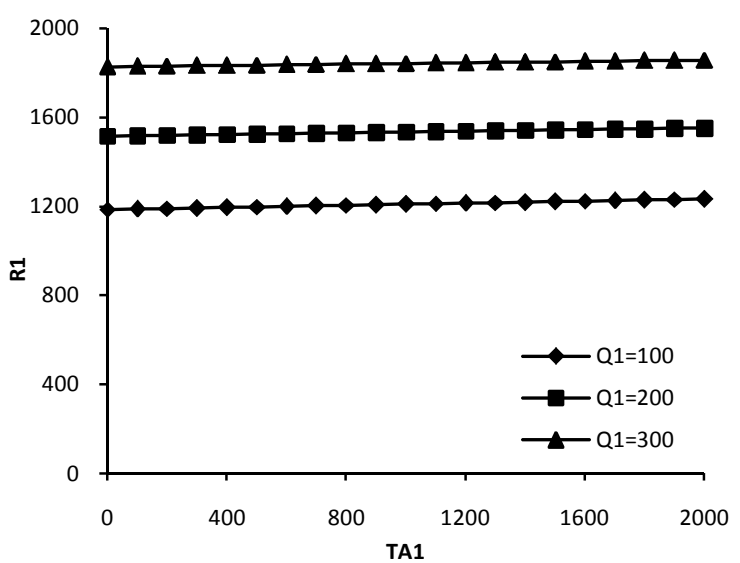

Figure 3. Variation of critical Rayleigh number $R_{1}$ with rotation parameter $T$ for a fixed $\in=0.5, P=0.005$ and $Q_{1}=100,200,300$.

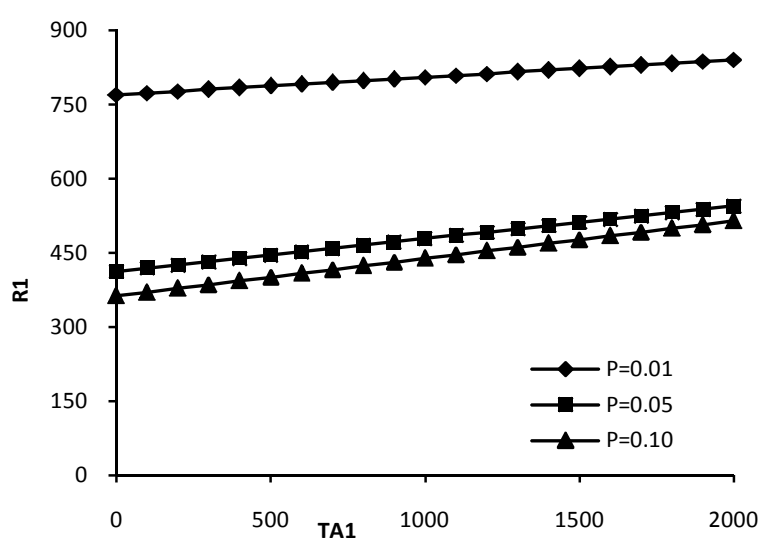

Figure 4. Variation of critical Rayleigh number $R_{1}$ with rotation parameter $T_{A_{1}}$ for a fixed $\in=0.5, Q_{1}=100$ and $P=0.01,0.05,0.10$.

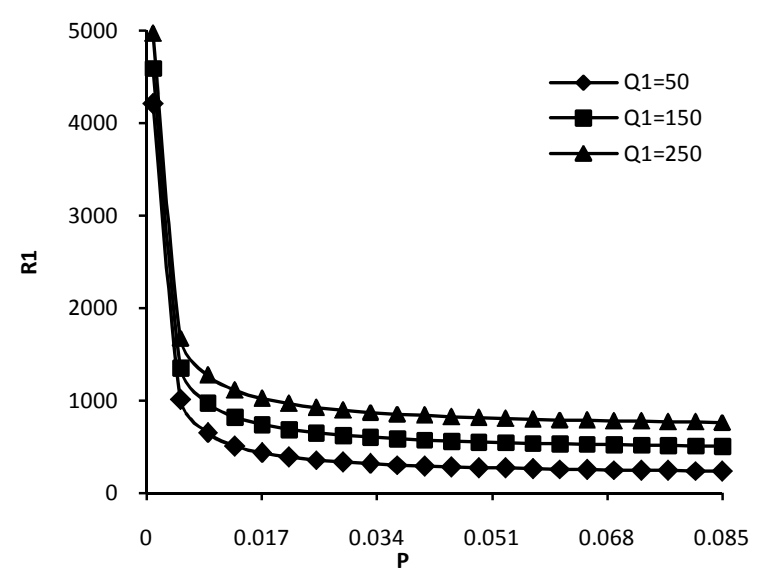

Figure 5. Variation of critical Rayleigh number $R_{1}$ with medium permeability $P$ for a fixed $\in=0.5, T_{A_{1}}=100$ and $Q_{1}=50,150,250$. 


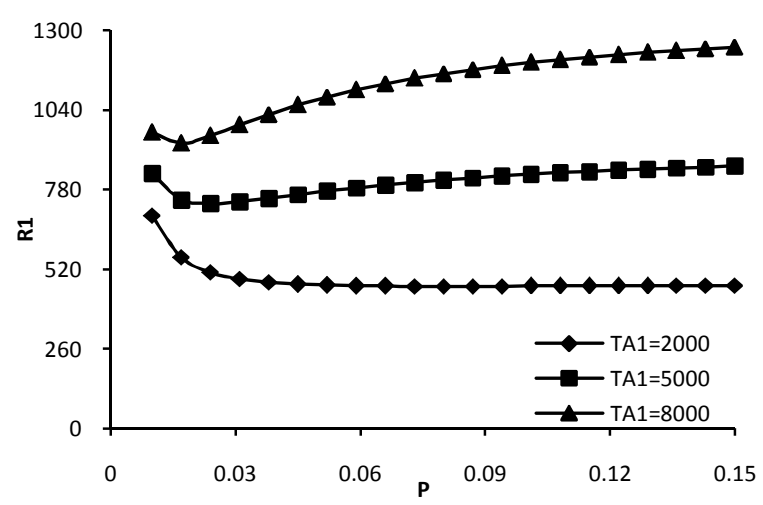

Figure 6. Variation of critical Rayleigh number $R_{1}$ with medium permeability $P$ for a fixed $\in=0.5, Q_{1}=50$ and $T_{A_{1}}=2000,5000,8000$.

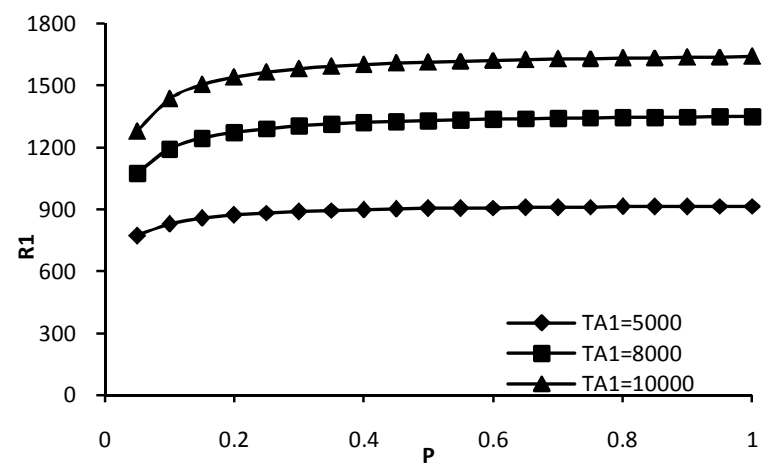

Figure 7. Variation of critical Rayleigh number $R_{1}$ with medium permeability $P$ for a fixed $\in=0.5, Q_{1}=50$ and $T_{A_{1}}=5000,8000,10000$.

\section{CONCLUSIONS}

With the growing importance of non-Newtonian fluids, magnetic field and rotation in chemical technology and industries, investigations on (Kuvshiniski-type) viscoelastic fluid are desirable. In the present paper, we have investigated the effect of magnetic field on a (Kuvshiniski-type) viscoelastic rotating fluid heated from below in porous medium. Dispersion relation governing the effects of magnetic field, rotation and medium permeability is derived. The main results from the analysis of the paper are as follow:

(i) For the case of stationary convection, a Kuvshiniski viscoelastic fluid behaves like an ordinary Newtonian fluid.

(ii) Magnetic field has a stabilizing effect on the thermal convection as is evident from the Equation (21) along with Figure 1 and Figure 2 for the permissible range of values of various parameters. In the absence of rotation, magnetic field clearly has a stabilizing effect on the thermal convection as is evident from Equation (22). Thus, the effect of increasing the magnitude of rotation parameter is to destabilize the system.

(iii) For the case of stationary convection, the rotation has a stabilizing effect on the thermal convection as can be seen from Equation (23), and graphically, from Figure 3 and Figure 4.

(iv) Medium permeability is found to have both stabilizing/destabilizing effects on the thermal convection as is evident from the Equation (24) along with Figure 5, Figure 6 and Figure 7 for the permissible range of values of various parameters. In the absence of rotation, medium permeability clearly has a destabilizing effect on the thermal convection as is evident from the Equation (25) as derived by Kumar and Singh [17]. In the absence of magnetic field medium permeability has both stabilizing/destabilizing effect on the thermal convection as is evident from the Equation (26) for the permissible range of values of various parameters as derived by Kumar and Singh [17].

(v) The oscillatory modes are introduced due to the presence of magnetic field and rotation in the system, whereas in their absence principle of exchange of stabilities (PES) is satisfied.

\section{ACKNOWLEDGEMENT}

The authors are grateful to the learned referees for their critical comments, which led to a significant improvement of the paper.

\section{REFERENCES}

1. Benard, H., "Les tourbillons cellulaires dans une nappe liquide", Rev. Gen. Sci. Pures Appl, Vol. 11, (1900), 1261-1271.

2. Rayleigh, L., " On convection currents in a horizontal layer of fluid, when the higher temperature is on the under side", The London, Edinburgh, and Dublin Philosophical Magazine and Journal of Science, Vol. 32, No. 192, (1916), 529-546.

3. Jeffreys, H., " The stability of a layer of fluid heated below", The London, Edinburgh, and Dublin Philosophical Magazine and Journal of Science, Vol. 2, No. 10, (1926), 833-844.

4. Chandrasekhar, S., "Hydrodynamic and hydromagnetic stability", Courier Dover Publications, (1961).

5. Bhatia, P. and Steiner, J., "Convective instability in a rotating viscoelastic fluid layer", ZAMM-Journal of Applied Mathematics and Mechanics/Zeitschrift für Angewandte Mathematik und Mechanik, Vol. 52, No. 6, (1972), 321-327.

6. Sharma, R., "Effect of rotation on thermal instability of a viscoelastic fluid", Acta Physica Academiae Scientiarum Hungaricae, Vol. 40, No. 1, (1976), 11-17.

7. Lapwood, E. R., "Convection of a fluid in a porous medium", Proceedings of the Cambridge, Philosophical Society, Vol. 44, (1948), 508-521.

8. Wooding, R. A., "Rayleigh instability of a thermal boundary layer in flow through a porous medium", Journal of Fluid Mechanics, Vol. 9, No. 02, (1960), 183-192. 
9. Beavers, G. S., Sparrow, E. M. and Magnuson, R. A., "Experiments on coupled parallel flows in a channel and a bounding porous medium", Journal of Fluids Engineering, Vol. 92, No.4, (1970), 843-848.

10. Joseph, D. and Tao, L., "The effect of permeability on the slow motion of a porous sphere in a viscous liquid", ZAMM-Journal of Applied Mathematics and Mechanics/Zeitschrift für Angewandte Mathematik und Mechanik, Vol. 44, No. 8-9, (1964), 361-364.

11. Saffman, P. G., "On the boundary conditions of the interface of a porous medium", Studies in Applied Mathematics, Vol. 1,No. 2, (1971), 93-101.

12. Lundgren, T. S., "Slow flow through stationary random beds and suspensions of spheres", Journal of fluid mechanics, Vol. 51, No. 02, (1972), 273-299.

13. Sharma, R.C., Sunil and Chand, S., "Thermosolutal instability of rivlin-ericksen rotating fluid in porous medium", Indian Journal of Pure and Applied Mathematics, Vol. 29, No. 4, (1998), 433-440.

14. Sharma, R.C., Sunil and Pal, M., "Thermosolutal convection in rivlin-ericksen rotating fluid in porous medium in hydromagnetics", Indian Journal of Pure and Applied Mathematics, Vol. 32, No. 1, (2001), 143-156.

15. Sharma, V. and Rana, G. C., "Thermal instability of a walters'(model B') elastico-viscous fluid in the presence of variable gravity field and rotation in porous medium", Journal of Non-Equilibrium Thermodynamics, Vol. 26, No. 1, (2001), 31-40.

16. Varshney, N. K. and Dwivedi, R. K., "Unsteady effect on mhd free convection and mass transfer flow of kuvshiniski fluid through porous medium with constant suction and constant heat and mass flux", Acta Ciencia Indica, Vol. 30, No. 2, (2004), 271-280.

17. Kumar, P. and Singh, M., "On a visco-elastic fluid heated from below in a porous medium", Journal of Non-Equilibrium Thermodynamics, Vol. 31, No. 2, (2006), 189-203.

18. Kumar, V. and Kumar, S., "On a couple-stress fluid heated from below in hydromagnetics", Applications and Applied Mathematics, Vol. 5, No. 10, (2010), 1529-1542.

19. Kumar, P., "Magneto-rotatory stability of two stratified kuvshiniski viscoelastic superposed fluids in porous medium", G. J. P. \& A. Sc. and Tech., Vol. 1, (2011), 28-35.

20. Bishnoi, J. and Goyal, N., "Soret dufour driven thermosolutal instability of darcy-maxwell fluid", International Journal of Engineering-Transactions A: Basics, Vol. 25, No. 4, (2012), $367-378$.

21. Joseph, D. D., "Stability of fluid motions", Springer-Verlag, Berlin, 1 \& 2, (1976).

\title{
Thermal Convection in a (Kuvshiniski-type) Viscoelastic Rotating Fluid TECHNICAL in the Presence of Magnetic Field through Porous Medium

\author{
V. Kumara, P. Kumarb \\ a Department of Mathematics, College of Engineering Studies, University of Petroleum \& Energy Studies (UPES), Dehradun-248007, \\ (Uttarakhand), India \\ ${ }^{b}$ Department of Mathematics, ICDEOL, Himachal Pradesh University, Shimla-171005, India
}

PAPER INFO

Paper history:

Received 22 July 2012

Received in revised form 10 December 2012 Accepted 28 February 2013

Keywords:

Thermal Convection

(Kuvshiniski-type) Viscoelastic Fluid

Magnetic Field

Rotation

Porous Medium

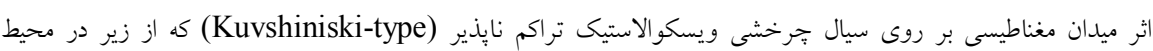

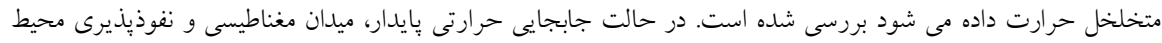

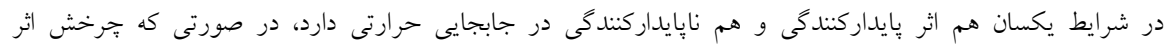

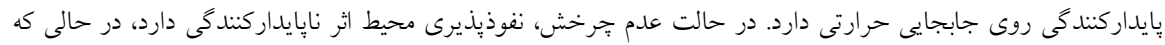

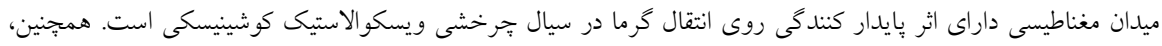

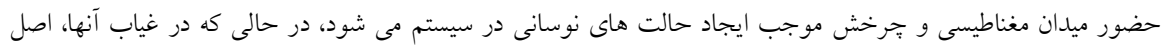

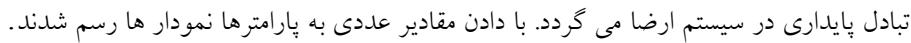

doi: 10.5829/idosi.ije.2013.26.07a.10 


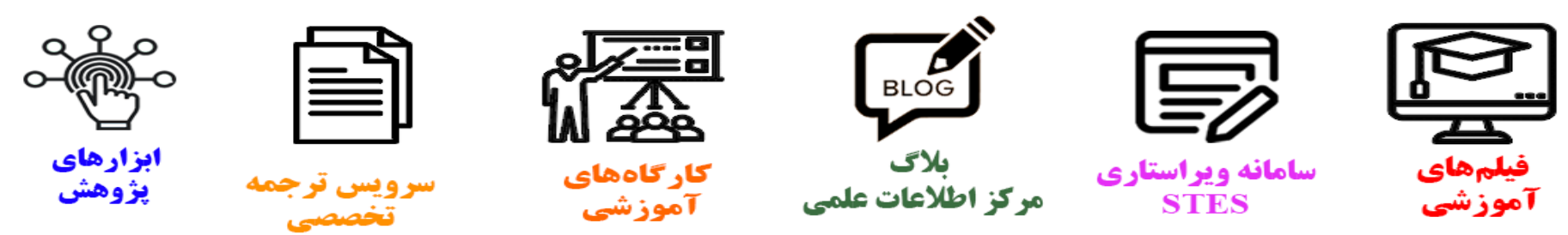

\section{(c)}

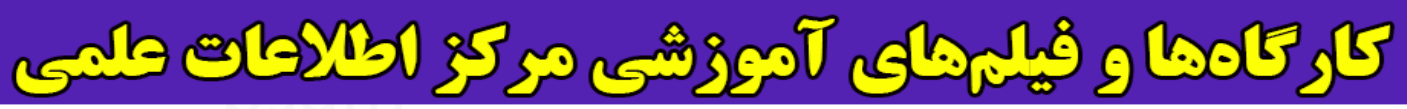
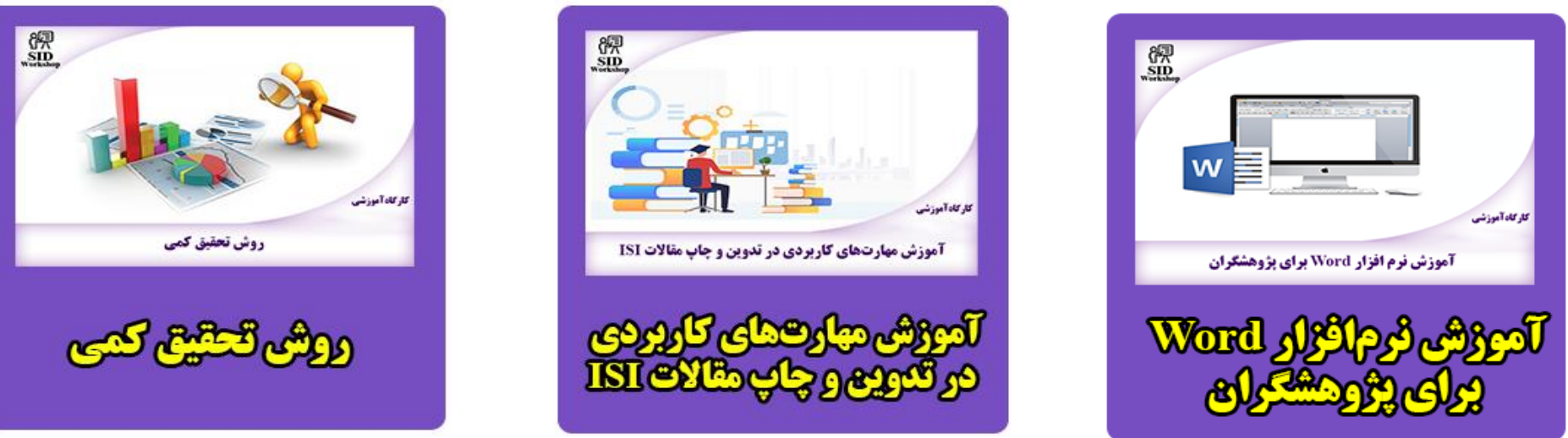